ARTICLE OPEN

\title{
Real-world retrospective cohort study ARCTIC shows burden of comorbidities in Swedish COPD versus non-COPD patients
}

\author{
Björn Ställberg ${ }^{1}$, Christer Janson ${ }^{2}$, Kjell Larsson ${ }^{3}$, Gunnar Johansson ${ }^{1}$, Konstantinos Kostikas ${ }^{4}$, Jean-Bernard Gruenberger ${ }^{4}$,
} Florian S. Gutzwiller ${ }^{4}$, Leif Jorgensen ${ }^{5}$, Milica Uhde ${ }^{6}$ and Karin Lisspers ${ }^{1}$

This study aimed to generate real-world evidence to assess the burden of comorbidities in COPD patients, to effectively manage these patients and optimize the associated healthcare resource allocation. ARCTIC is a large, real-world, retrospective cohort study conducted in Swedish COPD patients using electronic medical record data collected between 2000 and 2014 . These patients were studied for prevalence of various comorbidities and for association of these comorbidities with exacerbations, mortality, and healthcare costs compared with an age-, sex-, and comorbidities-matched non-COPD reference population. A total of 17,479 patients with COPD were compared with 84,514 non-COPD reference population. A significantly higher prevalence of various comorbidities was observed in COPD patients 2 years post-diagnosis vs. reference population, with the highest percentage increase observed for cardiovascular diseases ( $81.8 \%$ vs. $30.7 \%)$. Among the selected comorbidities, lung cancer was relatively more prevalent in COPD patients vs. reference population (relative risk, $\mathrm{RR}=5.97, p<0.0001$ ). Ischemic heart disease, hypertension, depression, anxiety, sleep disorders, osteoporosis, osteoarthritis, and asthma caused increased mortality rates in COPD patients. Comorbidities that were observed to be significantly associated with increased number of severe exacerbations in COPD patients included heart failure, ischemic heart disease, depression/anxiety, sleep disorders, osteoporosis, lung cancer, and stroke. The cumulative healthcare costs associated with comorbidities over 2 years after the index date were observed to be significantly higher in COPD patients $(€ 27,692)$ vs. reference population $(€ 5141)(p<0.0001)$. The data support the need for patient-centered treatment strategies and targeted healthcare resource allocation to reduce the humanistic and economic burden associated with COPD comorbidities.

npj Primary Care Respiratory Medicine (2018)28:33 ; doi:10.1038/s41533-018-0101-y

\section{INTRODUCTION}

Chronic obstructive pulmonary disease (COPD) is a common disease and it was estimated to affect 384 million persons in the year 2010, corresponding to a global prevalence of $11.7 \%$ (8.4\%-15.0\%). ${ }^{1}$ According to the 2010 Global Burden of Disease study, COPD has been estimated to be the third leading cause of mortality worldwide. ${ }^{2}$

COPD is also one of the leading causes of morbidity worldwide, and the morbidity is further affected by presence of concomitant chronic conditions. ${ }^{3}$ These chronic comorbidities play a potential role in the prognosis of COPD and health outcomes of patients. Evidence suggests that comorbidities increase the risk for exacerbations and hospitalizations, reduce health status, and increase the risk of mortality in COPD patients. ${ }^{4-11}$ Various comorbidities studied in patients with COPD include asthma; lung cancer; cardiovascular diseases, such as heart failure, ischemic heart disease, arrhythmias, peripheral vascular disease, and hypertension; osteoporosis; depression; anxiety; metabolic syndrome and diabetes; gastroesophageal reflux; bronchiectasis; and obstructive sleep apnea. ${ }^{3,5,8,12-16}$

Comorbidities are identified as major drivers of excess use of healthcare resource utilization (HCRU) and additional overall costs of managing COPD patients. ${ }^{6,17}$ A recently conducted study in the
Danish general practice settings reported that comorbidities cause up to $28 \%$ variation in the healthcare expenditures, whereas other demographic characteristics, such as age and sex only cause $5 \%$ variation. ${ }^{18}$ These healthcare expenditures further increase with increase in the number of comorbid conditions.

Hence, it is important to generate real-world evidence to better understand the burden of comorbidities in COPD to effectively manage these patients and find ways to optimize the associated healthcare resource allocation. The aim of this study was therefore to provide real-world evidence on the burden of comorbidities in COPD in the Swedish primary care and identify areas for improvement and management of COPD patients.

\section{RESULTS}

Out of the identified 18,586 eligible patients with a COPD diagnosis listed in EMRs, 291 patients were excluded as they were diagnosed with COPD before 40 years of age. Following case-control matching, a total of 17,479 patients with COPD (ICD10: J44.0) were studied under cases and these were compared with 84,514 age- and sex-matched control patients. Patient demographics of the COPD population and the age- and sex-matched reference population have been illustrated in the

\footnotetext{
${ }^{1}$ Department of Public Health and Caring Sciences, Family Medicine and Preventive Medicine, Uppsala University, Uppsala, Sweden; ${ }^{2}$ Department of Medical Sciences: Respiratory, Allergy and Sleep Research, Uppsala University, Uppsala, Sweden; ${ }^{3}$ Department of Pulmonary Medicine, Work Environment Toxicology, Karolinska Institutte, SE-171 77, Stockholm, Sweden; ${ }^{4}$ Novartis Pharma AG, Basel, Switzerland; ${ }^{5}$ IQVIA, Copenhagen, Denmark and ${ }^{6}$ IQVIA, Solna, Sweden Correspondence: Björn. Ställberg (b.stallberg@telia.com)
} 
Table 1. Age and sex distribution of patients with comorbidities in COPD and reference groups (age at index date and comorbidities 2 years before)

\begin{tabular}{|c|c|c|c|}
\hline Variable & $p$-value & $\begin{array}{l}\text { COPD population with } \\
\text { comorbidities }\end{array}$ & $\begin{array}{l}\text { Reference population } \\
\text { with comorbidities }\end{array}$ \\
\hline \multicolumn{4}{|l|}{ Heart failure } \\
\hline Age (years) & $<0.0001$ & $76.47(9.90)$ & 78.27 (9.99) \\
\hline Sex (F/M) & 0.0175 & $\begin{array}{l}1079(44.81) / 1329 \\
(55.19)\end{array}$ & $874(48.50) / 928(51.50)$ \\
\hline \multicolumn{4}{|c|}{ Ischemic heart disease } \\
\hline Age (years) & 0.1583 & $73.34(10.06)$ & $73.74(10.49)$ \\
\hline Sex (F/M) & 0.5396 & $\begin{array}{l}1063(42.98) / 1410 \\
(57.02)\end{array}$ & $\begin{array}{l}1261(42.16) / 1730 \\
(57.84)\end{array}$ \\
\hline \multicolumn{4}{|l|}{ Hypertension } \\
\hline Age (years) & 0.0007 & $71.15(10.07)$ & $70.54(10.68)$ \\
\hline $\operatorname{Sex}(F / M)$ & 0.0334 & $\begin{array}{l}2934(54.12) / 2487 \\
(45.88)\end{array}$ & $\begin{array}{l}4725(55.96) / 3718 \\
(44.04)\end{array}$ \\
\hline \multicolumn{4}{|c|}{ Depression/anxiety } \\
\hline Age (years) & $<0.0001$ & $65.60(12.01)$ & $63.62(12.15)$ \\
\hline Sex (F/M) & 0.5804 & $946(66.57) / 475(33.43)$ & $1406(67.47) / 678(32.53)$ \\
\hline \multicolumn{4}{|c|}{ Sleep disorders } \\
\hline Age (years) & 0.4421 & $64.52(9.34)$ & $64.02(9.82)$ \\
\hline $\operatorname{Sex}(F / M)$ & 0.0245 & $153(40.37) / 226(59.63)$ & $252(47.91) / 274(52.09)$ \\
\hline \multicolumn{4}{|c|}{ Osteoporosis } \\
\hline Age (years) & 0.6458 & $75.24(9.41)$ & $74.96(10.74)$ \\
\hline $\operatorname{Sex}(F / M)$ & 0.1146 & $449(87.18) / 66(12.82)$ & $550(90.16) / 60(9.84)$ \\
\hline \multicolumn{4}{|c|}{ Lung cancer } \\
\hline Age (years) & 0.2072 & $69.96(8.25)$ & $69.03(9.82)$ \\
\hline $\operatorname{Sex}(F / M)$ & 0.6146 & $214(50.00) / 214(50.00)$ & $111(52.11) / 102(47.89)$ \\
\hline \multicolumn{4}{|l|}{ Stroke } \\
\hline Age (years) & 0.3910 & 74.81 (9.99) & $75.29(10.31)$ \\
\hline Sex (F/M) & 0.4874 & $222(46.06) / 260(53.94)$ & $476(47.98) / 516(52.02)$ \\
\hline \multicolumn{4}{|l|}{ Osteoarthritis } \\
\hline Age (years) & $<0.0001$ & $70.82(10.03)$ & $68.20(10.50)$ \\
\hline Sex (F/M) & 0.0087 & $595(63.03) / 349(36.97)$ & $1943(67.68) / 928(32.32)$ \\
\hline \multicolumn{4}{|l|}{ Asthma } \\
\hline Age (years) & $<0.0001$ & $67.33(12.24)$ & $66.01(11.96)$ \\
\hline Sex (F/M) & 0.0407 & $\begin{array}{l}1681(61.60) / 1048 \\
(38.40)\end{array}$ & $\begin{array}{l}1817(64.25) / 1011 \\
(35.75)\end{array}$ \\
\hline
\end{tabular}

Mean (standard deviation) values presented for age, while $n$ (\%) values provided for gender

F/M females/males, COPD chronic obstructive pulmonary disease

recent publication on economic burden assessed in the ARCTIC study. $^{19}$

Patients in the COPD and reference populations with comorbidities, including hypertension, depression/anxiety, sleep disorders, lung cancer, osteoarthritis, and asthma were aged between 60 and 70 years, while populations with comorbidities, including heart failure, osteoporosis, and stroke had higher age distribution of $70-80$ years (Table 1). Although a similar age distribution was seen between the comorbidity and reference groups for most comorbidities, significant differences were observed among patients with heart failure, hypertension, depression/anxiety, osteoarthritis, and asthma (Table 1). Further, significant differences by sex were observed between the groups with heart failure, hypertension, sleep disorders, osteoarthritis, and asthma as comorbidities (Table 1).

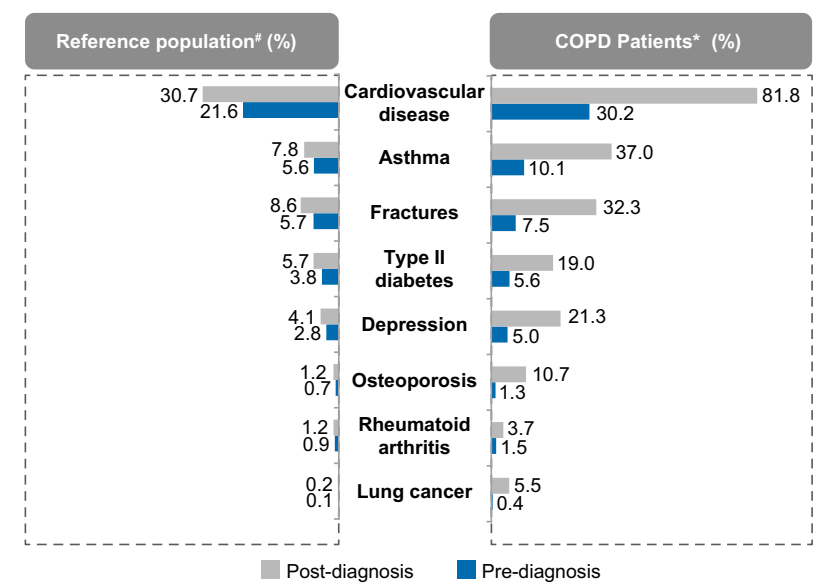

Fig. 1 Prevalence of comorbidities (\%) in COPD vs. reference population at 2 years pre- and post-first COPD diagnosis, $\# N=$ 52,$208 ;{ }^{*} N=13,052$. COPD chronic obstructive pulmonary disease

Prevalence of comorbidities

A significantly higher prevalence of the selected comorbidities was observed in COPD patients compared with the reference population at 2 years pre- and post-first COPD diagnosis $(p<$ 0.0001) (Fig. 1).

The prevalence of comorbidities increased in both groups after 2 years post-diagnosis. However, the percentage increase in the prevalence of comorbidities when calculated pre- and postdiagnosis was significantly higher in COPD patients compared with the reference population $(p<0.0001)$. The highest percentage increase was observed in the prevalence of cardiovascular diseases, which was $81.8 \%$ in COPD patients compared to $30.7 \%$ in the reference group (Fig. 1). The increase in the prevalence of comorbidities in the reference population might be attributed to the increase in age of the subjects.

Data showing $>5$-fold, $>3$-fold, and $>2.5$-fold increased prevalence of comorbidities in COPD population than the reference population are provided in the additional file (Tables S1, S2, and S3).

Among the nine selected comorbidities, lung cancer was more prevalent in patients with COPD than in the reference population (risk increased by 5.97), followed by heart failure (risk increased by 3.27) and osteoporosis (without current pathological fracture, risk increased by 2.75 and for osteoporosis with current pathological fracture, risk increased by 2.69) (Table 2).

Mortality

Comorbidities that led to significantly increased mortality in the COPD population than the reference population included ischemic heart disease, hypertension, depression, anxiety, sleep disorders, osteoporosis, osteoarthritis, and asthma. The highest risk of mortality was observed with sleep disorders (hazard ratio [HR]: 1.85 , 95\% confidence interval $[\mathrm{Cl}]$ : 1.34-2.56, $p=0.0002)$, followed by osteoarthritis (HR: $1.67,95 \%$ Cl: $1.44-1.93, p<0.0001$ ) (Table 3).

\section{Exacerbations}

Further, time to first exacerbation (any) in COPD patients was significantly decreased with the presence of the following comorbidities: heart failure, ischemic heart disease, hypertension, anxiety, osteoporosis, lung cancer, stroke, and asthma compared with COPD patients without these comorbidities (Table S4 in the additional file). Presence of lung cancer significantly decreased the time to first exacerbation, with a much larger magnitude compared with other comorbidities (HR: $1.56,95 \% \mathrm{Cl}$ : 1.40-1.73, 
Table 2. Percentage of patients with comorbidities in the case-control population ( 2 years before and after index date)

Comorbidities

Heart failure

Heart failure-150

Hypertensive heart disease-111

Ischemic heart disease

Angina pectoris-120

ST elevation (STEMI) and non-ST elevation (NSTEMI)

myocardial infarction-121

Chronic ischemic heart disease-I25

Hypertension

Essential (primary) hypertension-I10

Depression/anxiety

Major depressive disorder, single episode-F32

Major depressive disorder, recurrent-F33

Other anxiety disorders-F41

Sleep disorders

Sleep disorders-G47

Osteoporosis

Osteoporosis with current pathological fracture-M80

Osteoporosis without current pathological fracture-M81

Lung cancer

Malignant neoplasm of bronchus and lung-C34

Stroke

Cerebral infarction-163

Cerebral infarction-164

Osteoarthritis

Osteoarthritis of hip-M16

Osteoarthritis of knee-M17

Osteoarthritis of first carpometacarpal joint-M18

Other and unspecified osteoarthritis-M19

Others

Pulmonary embolism-126

Phlebitis and thrombophlebitis-180
COPD patients $(n=$ 17,479)

$5350(30.61)$

$456(2.61)$

$3308(18.93)$

2338 (13.38)

3448 (19.73)

$9838(56.28)$

3368 (19.27)

761 (4.35)

$2500(14.30)$

1439 (8.23)

809 (4.63)

1450 (8.30)

1091 (6.24)

$1782(10.20)$

$524(3.00)$

$1400(8.01)$

$1803(10.32)$

$443(2.53)$

1730 (9.90)

729 (4.17)

1140 (6.52)
Reference population $(n=$ 84,514 )

7899 (9.35)

903 (1.07)

7949 (9.41)

5026 (5.95)

7048 (8.35)

$31,675(37.51)$

8137 (9.63)

1676 (1.98)

5523 (6.54)

4039 (4.78)

1452 (1.72)

2552 (3.02)

883 (1.05)

5047 (5.98)

1496 (1.77)

$4710(5.58)$

$8573(10.15)$

1607 (1.90)

6331 (7.50)

1471 (1.74)

3450 (4.09)
3.27 (95\% Cl 3.17-3.37), $p<$ 0.0001

$2.44(95 \% \mathrm{Cl} 2.18-2.73), p<$ 0.0001

2.01 (95\% Cl 1.94-2.09), $p<$ 0.0001

2.25 (95\% Cl 2.15-2.35), $p<$ 0.0001

2.36 (95\% Cl 2.28-2.45), $p<$ 0.0001

1.50 (95\% Cl 1.48-1.52), $p<$ 0.0001

$2.00(95 \% \mathrm{Cl} 1.93-2.07), p<$ 0.0001

2.19 (95\% Cl 2.02-2.39), $p<$ 0.0001

2.19 (95\% Cl 2.09-2.29), $p<$ 0.0001

$1.72(95 \% \mathrm{Cl} 1.62-1.82), p<$ 0.0001

2.69 (95\% Cl 2.47-2.93), $p<$ 0.0001

2.75 (95\% Cl 2.58-2.92), $p<$ 0.0001

5.97 (95\% Cl 5.47-6.51), $p<$ 0.0001

$1.71(95 \% \mathrm{Cl} 1.62-1.80), p<$ 0.0001

1.69 (95\% Cl 1.53-1.87), $p<$ 0.0001

1.44 (95\% Cl 1.36-1.52), $p<$ 0.0001

$1.02(95 \% \mathrm{Cl} 0.97-1.07), p=$ 0.5037

1.33 (95\% Cl 1.20-1.48), $p<$ 0.0001

$1.32(95 \% \mathrm{Cl} 1.26-1.39), p<$ 0.0001

$2.39(95 \% \mathrm{Cl} 2.19-2.61), p<$ 0.0001

1.60 (95\% Cl 1.50-1.70), $p<$ 0.0001

ICD-10 codes mentioned alongside comorbidities

Cl confidence interval, COPD chronic obstructive pulmonary disease, ICD-10 International Classification of Diseases, tenth revision, NSTEMI non-ST-elevation myocardial infarction, STEMI ST-elevation myocardial infarction 
Table 3. Risk of mortality in COPD vs. reference populations with same comorbidities

\begin{tabular}{lll}
\hline Comorbidities & Hazard ratio $(95 \% \mathrm{Cl})$ & $p$-value \\
\hline Heart failure & $1.04(95 \% \mathrm{Cl} 0.97-1.13)$ & 0.2503 \\
Ischemic heart disease & $1.25(95 \% \mathrm{Cl} \mathrm{1.16-1.35)}$ & $<0.0001$ \\
Hypertension & $1.54(95 \% \mathrm{Cl} \mathrm{1.45-1.63)}$ & $<0.0001$ \\
Depression & $1.22(95 \% \mathrm{Cl} \mathrm{1.04-1.43)}$ & 0.0118 \\
Anxiety & $1.52(95 \% \mathrm{Cl} \mathrm{1.23-1.87)}$ & $<0.0001$ \\
Sleep disorders & $1.85(95 \% \mathrm{Cl} \mathrm{1.34-2.56)}$ & 0.0002 \\
Osteoporosis & $1.53(95 \% \mathrm{Cl} 1.27-1.83)$ & $<0.0001$ \\
Lung cancer & $0.69(95 \% \mathrm{Cl} 0.56-0.83)$ & 0.0001 \\
Stroke & $1.02(95 \% \mathrm{Cl} 0.89-1.18)$ & 0.7444 \\
Osteoarthritis & $1.67(95 \% \mathrm{Cl} 1.44-1.93)$ & $<0.0001$ \\
Asthma & $1.63(95 \% \mathrm{Cl} 1.47-1.81)$ & $<0.0001$ \\
\hline Hazard ratios reported for time to death between the two groups i.e., \\
COPD with comorbidity vs. reference population with comorbidity \\
Cl confidence interval, COPD chronic obstructive pulmonary disease \\
\hline
\end{tabular}

$p<0.0001$ ), followed by heart failure (HR: $1.40,95 \% \mathrm{Cl}: 1.33-1.47$, $p<0.0001$ ) (Table S4 in the additional file).

Comorbidities that were observed to be significantly associated with increased number of severe exacerbations in COPD patients included heart failure (rate ratio: $2.69,95 \% \mathrm{Cl}, 2.61-2.77$ ), ischemic heart disease (1.88, 95\% Cl: 1.83-1.93), depression/anxiety (1.44, $95 \% \mathrm{Cl}: 1.40-1.48)$, sleep disorders $(1.26,95 \% \mathrm{Cl}: 1.23-1.29)$, osteoporosis (1.53, 95\% Cl: 1.48-1.58), lung cancer $(1.32,95 \% \mathrm{Cl}$ : 1.17-1.47), and stroke (1.47, 95\% Cl: 1.42-1.52) (Table 4). Osteoarthritis was associated with lower number of severe exacerbations and hypertension had no effect on the number of severe exacerbations.

Costs

The cumulative HCRU costs over 2 years after the index date associated with comorbidities were significantly higher in COPD patients $(€ 27,692)$ compared with the reference group (€5141) ( $p$ $<0.0001$ ) (Fig. 2). Comorbidities accounted for $>80 \%$ of costs in COPD patients $(€ 22,292)$, with costs associated with hospital nights as the major contributor $(€ 18,879)$ when calculated 2 years after the index date (Fig. 2).

Table 4. Concurrent comorbidities and their association with the number of severe exacerbations

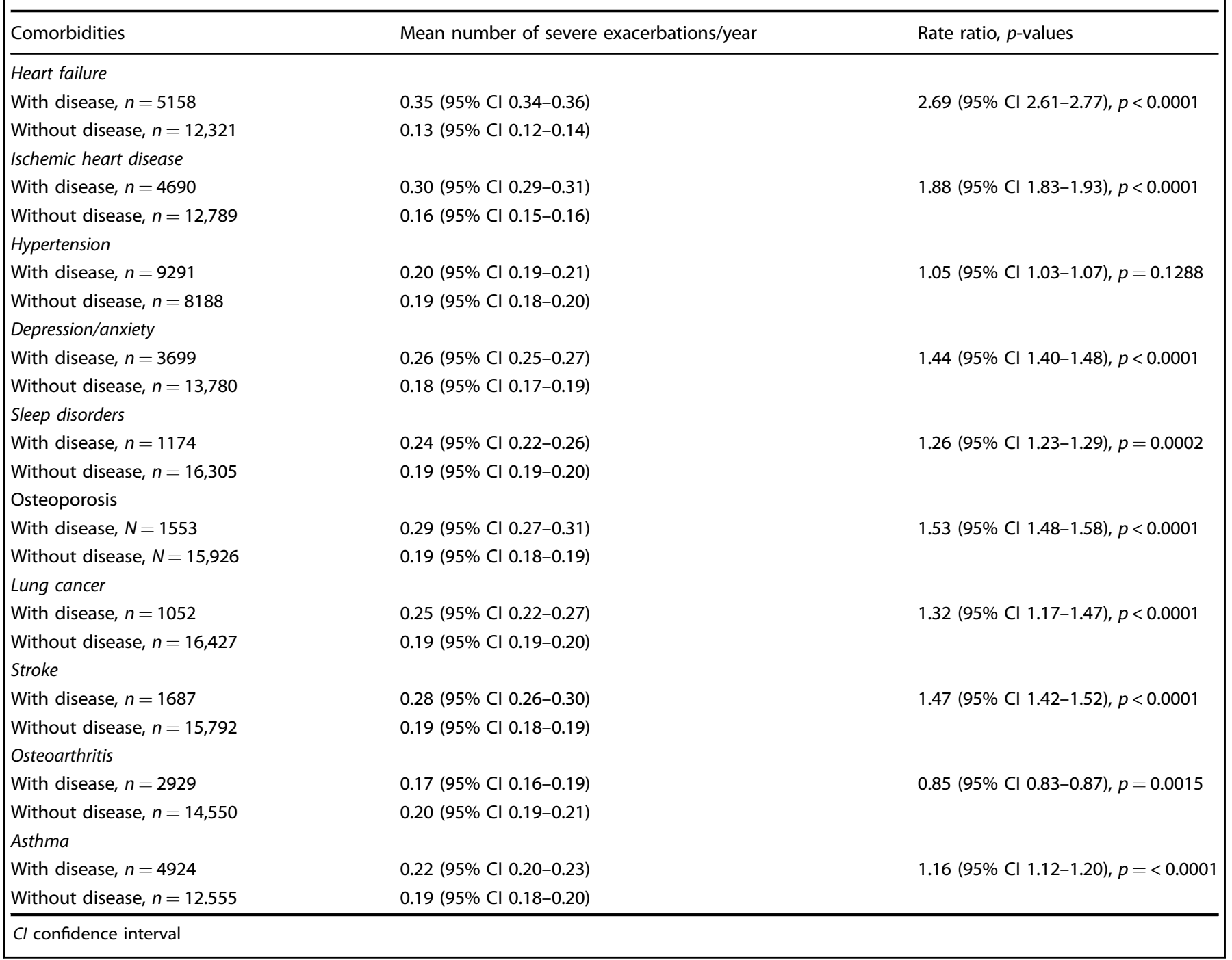




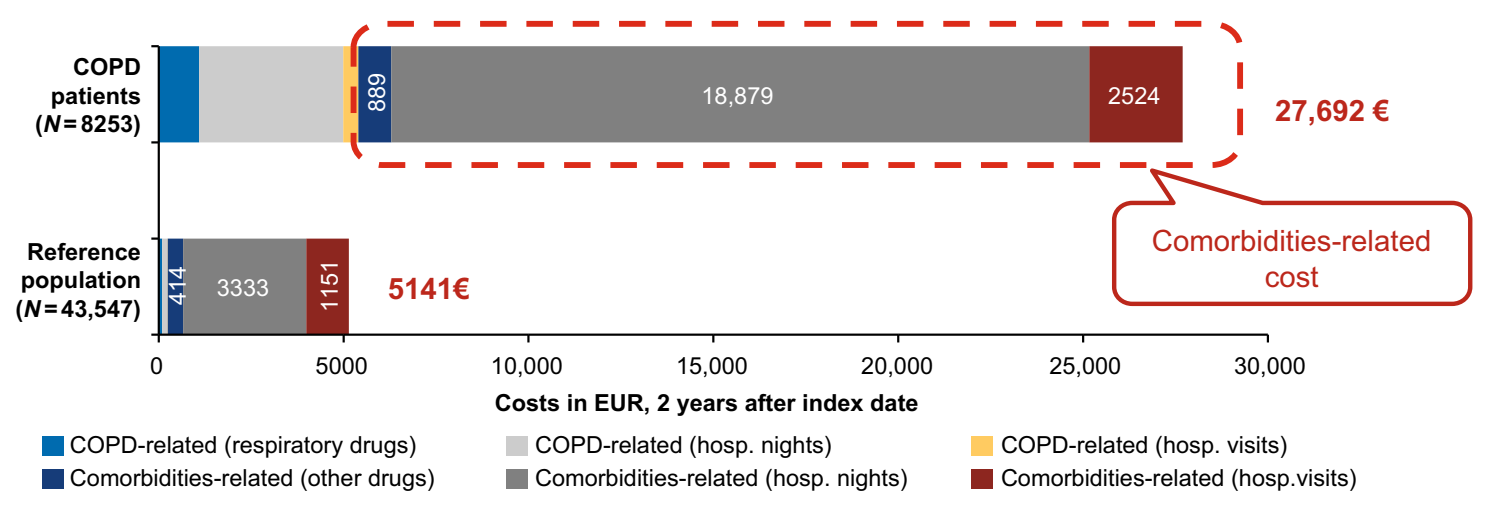

Fig. 2 HCRU in COPD patients vs. reference population-cumulative costs of 2 years after the index date, 2013, For the reference population, COPD-related costs include respiratory drugs mainly for asthma, COPD chronic obstructive pulmonary disease; EUR Euro; HCRU healthcare resource utilization

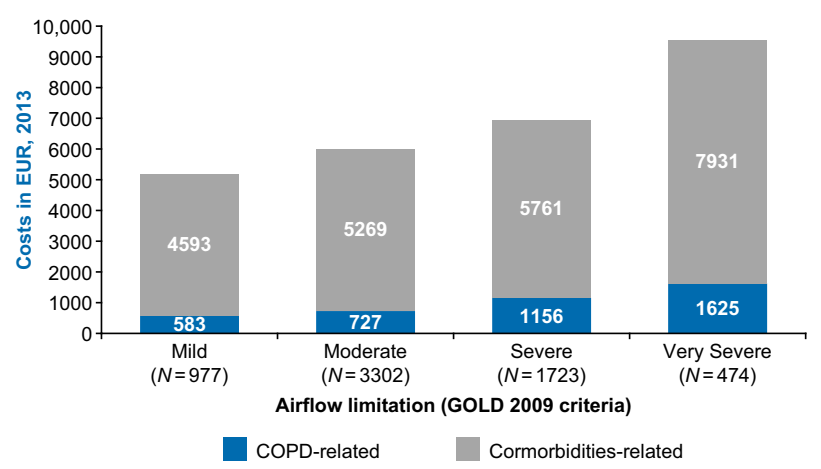

Fig. 3 HCRU in COPD patients based on severity strata cohort COPD chronic obstructive pulmonary disease; GOLD Global Initiative for Chronic Obstructive Lung Disease; HCRU healthcare resource utilization

Comorbidity-related costs were positively correlated with disease severity (mild vs. very severe accounted for €4593 vs. $€ 7931$, respectively) when analyzed in the COPD severity strata cohort (Fig. 3).

COPD patients aged between 60 and 65 years had the highest total costs associated with comorbidities, including sleep disorders ( $€ 41,751 /$ year; 43 times), osteoporosis ( $€ 50,234 /$ year; eight times), and heart failure (€36,465/year; three times) compared with the reference population with these comorbidities (Table S5 in the additional file).

Presence of more than one comorbidity

COPD patients with more than one comorbidity showed worse outcomes than those with only one comorbidity. COPD patients with both heart failure and diabetes $(n=3812)$, and both heart failure and angina $(n=3264)$ showed a considerably increased risk of mortality than the reference population with these comorbidities ( $N=5661$ and $N=3035$, respectively) (HR: $1.281,95 \% \mathrm{Cl}$ : 1.207-1.359, $p<0.0001$; HR: 1.167, 95\% Cl: 1.094-1.246, $p<0.0001$, respectively). Further, the time to first exacerbation was significantly decreased in COPD patients with both heart failure and diabetes, and both heart failure and angina compared to the COPD population without these comorbidities (HR: $1.306,95 \% \mathrm{Cl}$ : 1.255-1.360, $p<0.0001$; HR: 1.354, 95\% Cl: 1.297-1.414, $p<0.0001$, respectively). However, it was observed that having three comorbidities did not further increase the risk of mortality and exacerbations in COPD patients.

\section{DISCUSSION}

Main findings from this large, real-world retrospective study were that Swedish primary care patients with COPD had significantly higher prevalence of comorbidities compared with a non-COPD population. Prevalence rates of lung cancer, heart failure, ischemic heart disease, hypertension, depression/anxiety, sleep disorders, osteoporosis, stroke, osteoarthritis, and pulmonary embolism, were significantly higher in the COPD population compared with the non-COPD population. After 2 years post-diagnosis, the highest percentage increase was observed in the prevalence of cardiovascular diseases, which was $81.8 \%$ in COPD patients compared to $30.7 \%$ in the non-COPD population; this observation was in alignment with findings from other studies. ${ }^{12,14,15,20}$ Although aging increases the risk of developing several comorbidities, ${ }^{21}$ results from this study suggest that the presence of COPD significantly exaggerates the risk of such comorbidities in elderly patients.

Our results also indicated that these comorbidities have a high impact on the occurrence of severe exacerbations and mortality in COPD patients. It was found that COPD patients with heart failure, ischemic heart disease, depression/anxiety, sleep disorders, osteoporosis, lung cancer, and stroke were significantly more prone to have severe exacerbations than patients without these comorbidities. The reason why patients with osteoarthritis had fewer severe exacerbations remains unclear. Further, COPD patients with ischemic heart disease, hypertension, depression, anxiety, sleep disorders, osteoporosis, osteoarthritis, and asthma had a significantly higher risk of death than the non-COPD population. Research is needed though to establish cause of association of these comorbidities with the increased mortality rates in COPD patients. Surprisingly, a significantly lower risk of mortality was observed in COPD patients with lung cancer vs. the non-COPD population with lung cancer (HR: $0.69,95 \% \mathrm{Cl}$ : $0.56-0.83, p=0.0001)$. This might be due to earlier detection of lung cancer in COPD patients than in the non-COPD population as physicians expect lung cancer in COPD patients and often recommend them for X-ray imaging. The finding that relatively more frequent exacerbations and increased mortality were observed in COPD patients with more than one comorbidity indicates that exacerbations can also be prevented through proper management of comorbidities.

The cumulative healthcare costs associated with comorbidities were observed to be more than five times higher in the COPD population compared with the non-COPD population. Healthcare costs associated with these comorbidities were further found to increase with COPD severity.

The strength of this study is based on its large sample size, which comprised patients across the whole-COPD classification spectrum. Further, primary care setting and real-world study 
design represent the general population and clinical practice in Sweden. The study carried out a comprehensive assessment of comorbidities present in COPD patients and their association with exacerbations, mortality, and costs. Data quantification in this study allows decision-makers to identify-specific comorbidities that markedly increase burden among COPD patients and analyze how integrated screening and treatment strategies can be devised in such patients, thereby aiming to reduce the associated burden.

Results from this study are in agreement with other published studies; ${ }^{4-10,12-18,22-25}$ however, they provide more clear differentiation in terms of the risk association with exacerbations, mortality, and healthcare costs.

The study also has certain limitations. Because of the retrospective design of the study, the analysis could be affected by unobserved differences among patients. The reference population was identified from the study primary care centers rather than from the general population. Underestimation of comorbidities might have occurred, which is common in the elderly population. Further, the findings from this study are only limited to Sweden and cannot be generalized to populations applicable to other healthcare systems.

Nevertheless, the findings suggest that proper characterization of COPD patients needs to be carried out in terms of comorbidities as these are important prognostic variables that can significantly contribute to the increased morbidity and mortality in COPD patients. In addition, the results suggest the importance of patient-centered treatment strategies and the targeted allocation of healthcare resources in order to manage the associated humanistic and economic burden. ${ }^{26,27}$

In conclusion, the present study demonstrated a significantly increased prevalence of comorbidities in the COPD population than the non-COPD population. These comorbidities contribute to substantial morbidity, mortality, and economic burden in COPD patients. Therefore, these comorbidities should be comprehensively evaluated and considered while making clinical decisions in COPD patients.

\section{METHODS}

\section{Study design}

ARCTIC was a large, real-world, retrospective Swedish cohort study conducted in 18,586 eligible primary care COPD patients.

Electronic medical record (EMR) data collected between 2000 and 2014 for COPD patients from 52 primary care centers across Sweden using an established software system (Pygargus Customized eXtraction Program [CXP]) included age and gender, prescriptions (according to the World Health Organization Anatomic Therapeutic Chemical [ATC] codes), diagnoses (according to the International Classification of Diseases, tenth revision [ICD-10] codes), spirometry measurements, laboratory tests, healthcare professional (HCP) visits, and referrals. The centers covered urban and rural sites of varying sizes across Sweden. EMR data were linked using unique individual pseudonymized identification numbers to National Registry data sources. These included (i) the Longitudinal Integration Database for Health Insurance and Labor Market Studies, which contains socio-demographic data, including educational level, marital status and family situation, occupational status, retirement, and economic compensation and social benefits; (ii) the National Patient Register, which contains data relating to diagnosis from secondary care (ICD-10 code and associated position), including surgery, gender, age, region, hospital visits, specialty visits, hospital admissions, discharges, medical procedures, and surgeries performed in inpatient and outpatient specialist settings; (iii) the National Prescription Register, which contains the full details of all dispensed medications (ATC codes) from both primary and secondary care, including brand name, prescription date, dose, strength, pack size, and specialty of the prescriber and costs associated with the drug prescription; and (iv) the Cause of Death Register, which holds information relating to sex, date of death, and the underlying cause of death.

\section{Study patients}

The study included patients aged $\geq 40$ years who had received either a physician's diagnosis of COPD (ICD-10 code: J44) in primary care (EMR database) or a physician's diagnosis of asthma (ICD-10 code: J45/J46) in primary care that was later verified as COPD, or COPD was added to the asthma diagnosis in the hospital setting according to the National Patient Register. The index date was the time of the first recorded physician's diagnosis of COPD during the enrollment timeframe. A non-COPD reference population was identified at each center for age, sex, and matching comorbidities.

\section{Outcomes}

Various comorbidities assessed in the study are mentioned in the additional file. Chronic bronchitis was also analyzed; however, because of uncertainties in the usage of its diagnosis code, it was excluded. The comorbidity groups were created using comorbidity information at the COPD index date plus 6 months, since several comorbidities are diagnosed around the COPD index date.

Comorbidity prevalence (\%) of each disease at 2 years pre- and post-first COPD diagnosis and the associated impact on HCRU costs, including costs of hospital visits, hospital nights, and drugs were evaluated between COPD patients and non-COPD patients (reference population). Hospital nights were the sum of overnight stays at the hospital per patient. Hospitalization events were the number of times that a patient had been in a ward at a hospital. A visit to a hospital where the patients had not stayed overnight was considered to be a hospital visit. Costs were converted to the year 2013, and a conversion factor of 8/1 was used from Swedish Krona (SEK) to Euro $(€)$. Further, the association of these comorbidities with mortality and exacerbations was assessed. Exacerbations were defined as COPD-related hospitalizations (J44 in primary position or J44.0/J44.1 in secondary position), emergency visits (J44.0/J44.1 in outpatient hospital care), or collection of oral steroids (ATC H02AB) or antibiotics targeted at respiratory diseases (ATC J01AA/J01CA). Exacerbations occurring within 14 days were considered as one single event. Severe exacerbations were defined as hospitalizations due to COPD exacerbation in secondary care (ICD-10: J44) and/or emergency visits to a hospital for COPD (J44.1). Recurrent exacerbations occurring within 14 days were considered as one unique event. Within the COPD group, comorbidity-related costs were also analyzed based on the severity strata cohort (airflow limitation classified per the Global Initiative for Chronic Obstructive Lung Disease [GOLD] guidelines 2009: mild: $\mathrm{FEV}_{1} \geq 80 \%$ predicted, moderate: $50 \% \leq \mathrm{FEV}_{1}<80 \%$ predicted, severe: $30 \% \leq \mathrm{FEV}_{1}<50 \%$ predicted, and very severe: $\mathrm{FEV}_{1}<30 \%$ predicted or $\mathrm{FEV}_{1}<50 \%$ predicted plus chronic respiratory failure) and various age groups.

\section{Statistical analysis}

The time from the index date to the first exacerbation and time to death were compared between the comorbidity groups using Cox regression with time to event as the dependent variable, the comorbidity group as the independent variable, and age and gender as covariates. Hazard ratios between the groups were calculated along with $p$-values, comparing the differences between the groups. The effect of COPD on mortality was quantified by comparing the COPD population and the reference population for patients with the same comorbidity. Case-control cohorts were created for each comorbidity group between the COPD population and the reference population by matching age, sex, index year, and length of comorbidity duration. The index year was included to compensate for improved survival during the time period covered. An analysis of covariance was used to assess the association of comorbidities with the increased number of severe exacerbations. The number of severe exacerbations per year was accounted as the dependent variable, the comorbidity group as the independent variable, and age and gender as covariates. Indirect costs (defined as loss of income due to early retirement and sick leaves) were calculated for the COPD patients and compared across comorbidities. Differences between groups with and without the comorbidity were calculated and compared using an analysis of covariance with indirect cost as the dependent variable, groups with and without the comorbidity as independent variables, and age and gender as covariates.

Ethical approval for the original study was obtained from the local Ethical Regional Board in Uppsala, Sweden, on December 11, 2014 (number: 2014-397) for accessing the national health register and for recruiting primary care centers to the study. An amendment specifying additional analysis was approved by the Ethical Regional Board in Uppsala 
on October 6, 2017. All data were de-identified, and therefore patient consent was not required by the ethics committee.

\section{DATA AVAILABILITY}

The authors declare that data supporting the findings of this study are available within the paper and the additional file.

\section{ACKNOWLEDGEMENTS}

This study was funded by the Novartis Pharma AG (Basel, Switzerland). The authors would like to thank Purnima Pathak (Novartis) for managing and providing writing assistance in the development of this manuscript.

\section{AUTHOR CONTRIBUTIONS}

All authors participated equally in the study conception, design, statistical analysis' planning, analyzed, and interpreted the data and have been involved in reviewing and approving the manuscript.

\section{ADDITIONAL INFORMATION}

Supplementary information accompanies the paper on the npj Primary Care Respiratory Medicine website (https://doi.org/10.1038/s41533-018-0101-y).

Competing interests: B.S. has received honoraria for educational activities and lectures from AstraZeneca, Boehringer Ingelheim, Meda, Novartis and Teva, and has served on advisory boards arranged by AstraZeneca, Novartis, Meda, GlaxoSmithKline, Teva and Boehringer Ingelheim. K.K., J.-B.G., and F.S.G. are employed by Novartis Pharma AG, Basel, Switzerland. C.J. has received honoraria for educational activities and lectures from Novartis, AstraZeneca, GlaxoSmithKline, TEVA and Boehringer Ingelheim outside the submitted work. G.J. has participated in the steering committee by Novartis for this study and served on advisory boards arranged by AstraZeneca, Novo Nordisk, and Takeda. K.L. has, during the last 5 years, on one or more occasion served in an advisory board and/or served as speaker and/or participated in education arranged by AstraZeneca, Boehringer Ingelheim, Chiesi, GlaxoSmithKline, Orion, Novartis, TEVA and Takeda. L.J. and M.U. are employed by IQVIA. K.L. has received payments for educational activities and lectures from AstraZeneca, GlaxoSmithKline, Meda, MSD, Novartis and Nycomed, served on advisory boards arranged by Novartis and Meda, and has participated in the steering committee by Novartis for this study.

Publisher's note: Springer Nature remains neutral with regard to jurisdictional claims in published maps and institutional affiliations.

\section{REFERENCES}

1. Adeloye, D. et al. Global and regional estimates of COPD prevalence: systematic review and meta-analysis. J. Glob. Health 5, 020415 (2015).

2. Lozano, R. et al. Global and regional mortality from 235 causes of death for 20 age groups in 1990 and 2010: a systematic analysis for the Global Burden of Disease Study 2010. Lancet 380, 2095-2128 (2012).

3. GOLD. Global strategy for the diagnosis, management, and prevention of chronic obstructive pulmonary disease, http://goldcopd.org/ (2017).

4. Westerik, J. A. M. et al. Associations between chronic comorbidity and exacerbation risk in primary care patients with COPD. Respir. Res. 18, 31 (2017).

5. Gudmundsson, G. et al. Depression, anxiety and health status after hospitalisation for COPD: a multicentre study in the Nordic countries. Respir. Med. 100, 87-93 (2006).

6. Terzano, C. et al. Comorbidity, hospitalization, and mortality in COPD: results from a longitudinal study. Lung 188, 321-329 (2010).

7. Putcha, N., Drummond, M. B., Wise, R. A. \& Hansel, N. N. Comorbidities and chronic obstructive pulmonary disease: prevalence, influence on outcomes, and management. Semin. Respir. Crit. Care Med. 36, 575-591 (2015).

8. Sundh, J. et al. Comorbidity and health-related quality of life in patients with severe chronic obstructive pulmonary disease attending Swedish secondary care units. Int. J. Chronic Obstr. Pulm. Dis. 10, 173-183 (2015).
9. Papaioannou, A. I. et al. Cardiovascular comorbidities in hospitalised COPD patients: a determinant of future risk? Eur. Respir. J. 46, 846-849 (2015).

10. Jencks, S. F., Williams, M. V. \& Coleman, E. A. Rehospitalizations among patients in the medicare fee-for-service program. New Engl. J. Med. 360, 1418-1428 (2009).

11. Johansson, G. et al. Exacerbations and healthcare resource utilization among COPD patients in a Swedish registry-based nation-wide study. BMC Pulm. Med. 18, 17 (2018)

12. Chen, W., Thomas, J., Sadatsafavi, M. \& FitzGerald, J. M. Risk of cardiovascular comorbidity in patients with chronic obstructive pulmonary disease: a systematic review and meta-analysis. Lancet Respir. Med. 3, 631-639 (2015).

13. Fabbri, L. M., Luppi, F., Beghe, B. \& Rabe, K. F. Complex chronic comorbidities of COPD. Eur. Respir. J. 31, 204-212 (2008).

14. Mannino, D. M., Thorn, D., Swensen, A. \& Holguin, F. Prevalence and outcomes of diabetes, hypertension and cardiovascular disease in COPD. Eur. Respir. J. 32, 962-969 (2008).

15. Soriano, J. B., Visick, G. T., Muellerova, H., Payvandi, N. \& Hansell, A. L. Patterns of comorbidities in newly diagnosed COPD and asthma in primary care. Chest $\mathbf{1 2 8}$, 2099-2107 (2005).

16. Divo, M. et al. Comorbidities and risk of mortality in patients with chronic obstructive pulmonary disease. Am. J. Respir. Crit. Care Med. 186, 155-161 (2012).

17. Mannino, D. M. et al. Economic burden of COPD in the presence of comorbidities. Chest 148, 138-150 (2015).

18. Ahnfeldt-Mollerup, P., Lykkegaard, J., Halling, A., Olsen, K. R. \& Kristensen, T. Resource allocation and the burden of co-morbidities among patients diagnosed with chronic obstructive pulmonary disease: an observational cohort study from Danish general practice. BMC Health Serv. Res. 16, 121 (2016).

19. Lisspers, K. et al. Economic burden of COPD in a Swedish cohort: the ARCTIC study. Int J. Chron. Obstruct Pulmon Dis. 13, 275-285 (2018).

20. Janson, $C$. et al. Characteristics of hospitalised patients with COPD in the Nordic countries. Respir. Med. 100, S10-S16 (2006).

21. Divo, M. J., Martinez, C. H. \& Mannino, D. M. Ageing and the epidemiology of multimorbidity. Eur. Respir. J. 44, 1055-1068 (2014).

22. Baty, F., Putora, P. M., Isenring, B., Blum, T. \& Brutsche, M. Comorbidities and burden of COPD: a population based case-control study. PLOS ONE 8, e63285 (2013).

23. Dal Negro, R. W., Bonadiman, L. \& Turco, P. Prevalence of different comorbidities in COPD patients by gender and GOLD stage. Multidiscip. Respir. Med. 10, 24 (2015).

24. Terzano, C. et al. Chronic obstructive pulmonary disease (COPD) exacerbation: impact of comorbidities on length and costs during hospitalization. Eur. Rev. Med. Pharmacol. Sci. 21, 3680-3689 (2017).

25. Calverley, P. M. et al. Cardiovascular events in patients with COPD: TORCH study results. Thorax 65, 719-725 (2010).

26. Luppi, F., Franco, F., Beghe, B. \& Fabbri, L. M. Treatment of chronic obstructive pulmonary disease and its comorbidities. Proc. Am. Thorac. Soc. 5, 848-856 (2008).

27. Chatila, W. M., Thomashow, B. M., Minai, O. A., Criner, G. J. \& Make, B. J. Comorbidities in chronic obstructive pulmonary disease. Proc. Am. Thorac. Soc. 5 549-555 (2008).

Open Access This article is licensed under a Creative Commons Attribution 4.0 International License, which permits use, sharing, adaptation, distribution and reproduction in any medium or format, as long as you give appropriate credit to the original author(s) and the source, provide a link to the Creative Commons license, and indicate if changes were made. The images or other third party material in this article are included in the article's Creative Commons license, unless indicated otherwise in a credit line to the material. If material is not included in the article's Creative Commons license and your intended use is not permitted by statutory regulation or exceeds the permitted use, you will need to obtain permission directly from the copyright holder. To view a copy of this license, visit http://creativecommons. org/licenses/by/4.0/.

(c) The Author(s) 2018 